\title{
Permanent Magnet Synchronous Motor Direct Torque Control Based on Stator Resistance Online Estimation
}

\author{
Du Xue, Zhang Suying, Jin Ji, Zhao Peidi, Liu Huixian
}

School of Electrical Engineering, Hebei University of Science and Technology, Shijiazhuang, China

Email address:

duxue_xdd@163.com (Du Xue),923252999@qq.com (Zhang Suying),651419346@qq.com (Jin Ji)

\section{To cite this article:}

Du Xue, Zhang Suying, Jin Ji, Zhao Peidi, Liu Huixian. Permanent Magnet Synchronous Motor Direct Torque Control Based on Stator Resistance Online Estimation. Science Discovery. Vol. 5, No. 1, 2017, pp. 33-39. doi: 10.11648/j.sd.20170501.16

Received: March 10, 2017; Accepted: April 6, 2017; Published: April 12, 2017

\begin{abstract}
Permanent magnet synchronous motor direct torque control system is characterized by its fast, automatic control and so on. Based on the actual value and reference value of the motor control system, this article analysis the effect of the stator resistance variation on the performance of the system.A method to track stator resistance variation is proposed according to the effect of stator resistance deviation on stator flux and current vector.Based on the optimal reference value of the stator current vector, the stator resistance value is estimated on-line by using the angle between the electromagnetic torque and the stator flux; and the simulation studies results using Matlab/Simulink shows that the control method is very effective to improve the low-speed performance of the system, reduced torque ripple and improve system robustness.
\end{abstract}

Keywords: PMSM, DTC, Online Stator Resistance Estimation, Robustness

\section{基于定子电阻在线估计的永磁同步电机 直接转矩控制系统性能研究}

杜雪，张苏英，金吉，赵培迪，刘慧贤

电气工程学院, 河北科技大学, 石家庄, 中国

邮箱

duxue_xdd@163.com（杜雪），923252999@qq. com（张苏英），651419346@qq.com（金吉）

摘要: 永磁同步电机直接转矩控制系统以其快速性、自控性等特点著称。文章从电机控制系统的实际值和参考值出发, 分析定子电阻变化对系统性能的影响, 针对定子电阻误差对定子磁链和电流矢量的影响, 提出一种跟踪定子电阻变化的 方法。系统以定子电流矢量参考值最优为出发点, 利用电磁转矩与定子磁链之间夹角对定子电阻值进行在线估计, 并用 Matlab/Simulink进行仿真研究, 结果表明: 该控制方法很好的改善系统的低速性能, 降低转矩脉动提高系统鲁棒性。

关键词：永磁同步电机, 直接转矩控制, 定子电阻在线估计, 鲁棒控制

\section{1. 引言}

永磁同步电机 (Permanent Magnet Synchronous Motor-PMSM）以其高效性、高能量密度以及高转矩惯量比
得到了诸多关注，广泛应用于轨道交通、医疗设备、石油 工业、家用电器等领域 [1-3]。直接转矩控制 (Direct Torque Control-DTC) 是继矢量控制之后出现的一直交流电机调速 控制方案 [4]。通常DTC系统计算在静止坐标系中进行, 定 子磁链通过定子电阻观测得到, 不随转子参数的变化而变 
化, 具有鲁棒性好、效率高等特点 $[5-6]$ 。当电机在高速 运行时, 定子电阻压降相对系统定子磁链影响较小, 可忽 略不计, 因而高速时动态性能良好。电机在低速运行时, 定子电阻的压降分量增大, 定子磁链发生变化致使开关区 间判断错误, 严重影响了电磁转矩的动态性能 [7-9]。考 虑到定子电阻阻值对DTC系统性能的重要性, 越来越多的 学者研究定子电阻在线估计。目前比较经济的方法是通过 参考模型计算得到定子电阻 [10-12]。文献 [13]所采用的 阻值是采用自适应PI调节器进行在线估计。文献 [14] 中采 用电流模型对定子磁链进行相关修正。文献 [15-18]对此 分别提出了改进方法, 例如神经网络、小波分析等方法具 有一定的参考意义, 但还存在若干不足。由于电机在低速 运行时还有许多较为复杂的问题。本文在传统DTC基础上, 提出基于定子电阻在线估计的永磁同步电机直接转矩控 制, 通过实时检测定子电流, 准确计算定子磁链, 减小转 矩脉动的同时提高系统低速性能。

\section{2. 永磁同步电机的数学模型}

PMSM是一个强耦合、复杂的非线性系统, 为了能够更 好地设计先进的PMSM控制算法, 建立合适的数学模型, 假 设PMSM为理想电机，做了以下几点假设 $[11,19]$ :

1）忽略磁场的高次谐波, 在气隙中永磁体产生的磁 场和电机定子电流产生的磁场均为正弦分布;

2) 忽略磁滞和浴流损耗的影响, 以及定、转子铁芯 磁阻;

3）定子绕组三相对称, 空间上各绕组轴线互差 $120^{\circ}$ 电角度;

4）忽略电机永磁体的阻尼作用。

在对PMSM的研究中, $i_{A}, i_{B}, i_{C}$ 与 $i_{\alpha}, i_{\beta}$ 之间, $i_{\alpha}, i_{\beta}$ 与 $i_{d}, i_{q}$ 之间变量存在耦合。为更好更快的实现解耦, 为便于计算 研究, 专家和学者们引入空间矢量坐标系的概念。PMSM 数学模型如图1所示。

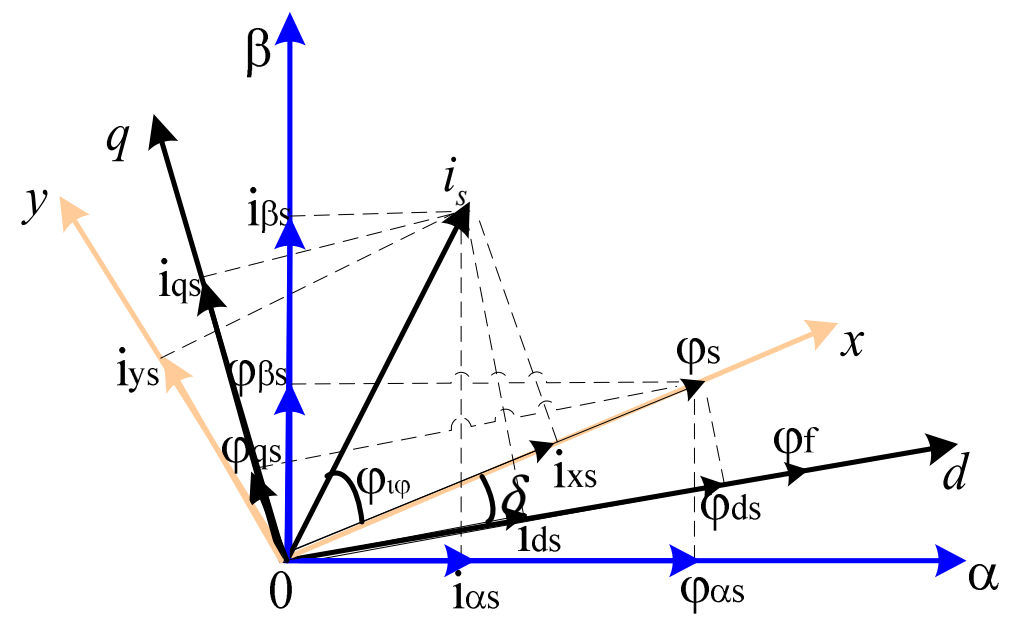

图1 两相坐标系。

在 $d-\mathrm{q}$ 坐标下, PMSM数学建模如下:

$$
\begin{gathered}
\left\{\begin{array}{l}
u_{d}=\frac{d \psi_{d}}{d t}-\psi_{q} \omega_{r}+R_{s} i_{d} \\
u_{q}=\frac{d \psi_{q}}{d t}-\psi_{d} \omega_{r}+R_{s} i_{q}
\end{array}\right. \\
\left\{\begin{array}{l}
\psi_{d}=L_{d} i_{d}+\psi_{f} \\
\psi_{q}=L_{q} i_{q}
\end{array}\right. \\
T_{e}=\frac{3}{2} p\left(\psi_{d} i_{q}-\psi_{q} i_{d}\right)=\frac{3}{2} p\left[\psi_{f} i_{q}+\left(L_{d}-L_{q}\right) i_{d} i_{q}\right]
\end{gathered}
$$

在 $\alpha-\beta$ 两相静止坐标系下, PMSM定子磁链和转矩方 程如下:

$$
\left\{\begin{array}{l}
\psi_{\alpha}=\int\left(u_{\alpha}-R_{s} i_{\alpha}\right) d t \\
\psi_{\beta}=\int\left(u_{s \beta}-R_{s} i_{\beta}\right) d t
\end{array}\right.
$$

$$
T_{e}=\frac{3}{2} p\left(\psi_{\alpha} i_{\beta}-\psi_{\beta} i_{\alpha}\right)
$$

式中: $u_{d}, u_{q}---$ 定子电压在 $d-q$ 轴上的分量; $i_{d}, i_{q}$ ---定子电流在 $d-q$ 轴上的分量; $\psi_{d}, \psi_{q}--$ 定子磁链在 $d-q$ 轴上的分量; $L_{d}, L_{q}---$ 定子绕组在 $d-q$ 轴上的电感; $u_{\alpha}, u_{\beta}--$ 定子电压在 $\alpha-\beta$ 轴上的分量, $i_{s \alpha}, i_{s \beta}--$ 定子电 流在轴上的分量, $\psi_{s \alpha}, \psi_{s \beta}--$ 定子磁链在 $\alpha-\beta$ 轴上的分 量, $R_{s}, \omega_{r}, \psi_{f}---$ 分别为定子电阻, 转子角速度, 转子永 磁体磁链; $T_{e}$---电机电磁转矩; $p$--电机转子极对数;

\section{3. 永磁同步电机定子电阻设计}

\section{1. 定子电阻对系统性能的影响}

永磁同步电机DTC控制回路中仅需要两个滞环控制器 和一个转速环PI调节器, 电机在运行时, 将逆变器和电机 作为一个整体, DTC的运算均在静止坐标系中进行, 简化了 
电机的数学模型, 同时大大降低了控制系统对电机参数的 依赖性。电机运行时, 通过实时检测电机定子电压和电流, 计算转矩和磁链的幅值, 并分别与转矩和磁链的给定值进 行比较, 利用所得差值来控制定子磁链的幅值及该矢量相
对于磁链的夹角, 由转矩和磁链调节器直接输出所需的空 间电压矢量, 从而达到磁链和转矩直接控制的目的 [20-21]。永磁同步电机直接转矩控制系统结构框图如图2 所示。

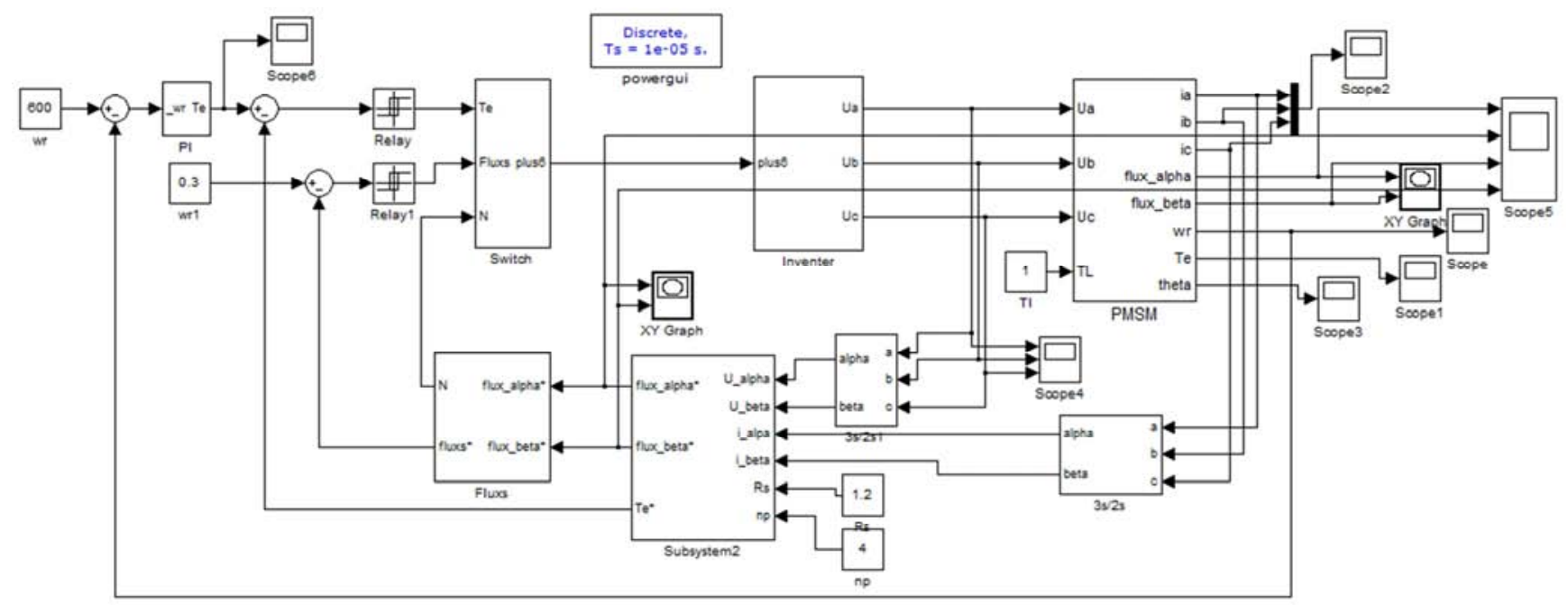

图2 永磁同步电机直接转矩控制系统结构框图。

一个完整电机直接转矩控制系统的运行需要对定子 磁链和电磁转矩的估计有一个满意的性能, 从而减小系统 稳态误差。假定控制系统在电机运行时不受定子电阻影响, 那么电机运行时实际的定子磁链 $\phi_{s}$ 、估计定子磁链 $\phi_{s c}$ 、 参考磁链 $\phi_{s}^{*}$ 为:

$$
\phi_{s}^{*}=\phi_{s}=\phi_{s c}=\int\left(u_{s}-R_{s} i_{s}\right) d t+\left.\phi_{s}\right|_{t=0}
$$

参考转矩 $T e_{s}^{*}$ 、实际转矩 $T e$ 、估计转矩 $T e_{c}$ 为:

$$
T_{e}^{*}=T_{e}=T_{e c}=\frac{3}{2} p\left|\phi_{s} \times i_{s}\right|
$$

电机在实际运行过程中由于温升和电机开关频率的 变化, 定子电阻会产生相应变化。即:

$$
R_{s}=R_{s c}+\Delta R
$$

上式中, $R_{s}, R_{s c}, \Delta R$ 分别代表实际电阻, 估计电阻和 电阻误差。根据小波信号分析, 定子电阻误差可分为两部 分, 其中消极的定子电阻误差使DTC系统性能变差; 然而 积极的误差信号能够调节实际电阻和估计电阻之间的差 别, 起到有利的作用。电机运行时估计磁链估计转矩分别 为:

$$
\begin{gathered}
\phi_{s c}=\int\left(u_{s}-R_{s} i_{s}\right) d t+\left.\phi_{s}\right|_{t=0} \\
T_{e c}=\frac{3}{2} p\left|\phi_{s c} \times i_{s}\right|
\end{gathered}
$$

将式（8）代入式（9）中得:

$$
\phi_{s c}=\phi_{s}+\int_{t=0}^{t}\left(\Delta R_{s} i_{s}\right) d t=\phi_{s}+\frac{1}{j \omega} \Delta R_{s} i_{s}
$$

上式中 $\omega$ 是旋转速度, 将上式 (11) 代到式 (10) 中 得:

$$
T_{e c}=T_{e}+\frac{3 p}{2 \omega} \Delta R_{s}\left|i_{s}\right|^{2}
$$

结合 (6) (12) 得:

$$
\begin{gathered}
\left|\phi_{s}\right|=\sqrt{\left|\phi_{s}^{*}\right|^{2}+\left(\frac{\Delta R_{s}\left|i_{s}\right|}{\omega}\right)^{2}-2\left|\phi_{s}^{*}\right| \Delta R_{s} \frac{\left|i_{s}\right| \sin \left(\phi_{i \phi_{c}}\right)}{\omega}} \\
T_{e}=T_{e}^{*}-\frac{3 p}{2 \omega} \Delta R_{s}\left|i_{s}\right|^{2}
\end{gathered}
$$

其中, $\phi_{i \phi c}$ 为定子磁链和电流矢量之间的夹角估计值。 由（13）和 (14) 知, 系统在低速运行时, 受定子电阻变 化的影响, 电磁转矩和定子磁链都会发生相应的改变。

\section{2 . 定子电阻估计器的设计}

在DTC系统中，定子磁链和电流矢量的参考值都是依 据输入转矩和磁链计算得到的。根据图 1 , 在 $\mathrm{d}-\mathrm{q}$ 坐标系下 有:

$$
\left\{\begin{array}{l}
\phi_{d s}=\left|\phi_{s}\right| \cos \delta \\
\phi_{q s}=\left|\phi_{s}\right| \sin \delta \\
\tan \left(\phi_{i \phi}\right)=\frac{T_{e}}{T_{r}}
\end{array}\right.
$$




$$
T_{e}=\frac{3}{2} p\left|\phi_{s}\right|\left|i_{s}\right| \sin \left(\phi_{i \phi}\right)=\frac{3}{2} p\left(\psi_{d s} i_{q s}-\psi_{q s} i_{d s}\right)
$$

式中 $\delta$ 为转矩角。将式 (2) (15) 代入式 (16) 得:

$$
T_{e}=\frac{3}{2} p\left|\phi_{s}\right|\left(\frac{\phi_{f}}{L_{d}} \sin \delta+\frac{\left|\phi_{s}\right|}{2}\left(\frac{1}{L_{q}}-\frac{1}{L_{d}}\right) \sin 2 \delta\right)
$$

同理, 得无功转矩:

$$
\begin{aligned}
T_{r} & =\frac{3}{2} p\left|\phi_{s}\right|\left|i_{s}\right| \cos \left(\phi_{i \phi}\right) \\
& =\frac{3}{2} p\left|\phi_{s}\right|\left(\frac{\left|\phi_{s}\right|}{L_{d}} \cos ^{2} \delta+\frac{\left|\phi_{s}\right|}{L_{q}} \sin ^{2} \delta-\frac{\phi_{f}}{L_{d}} \cos \delta\right)
\end{aligned}
$$

根据式（15）（18）, 我们得到电机运行时转矩, 电流矢量等相关参考值为:

$$
\begin{gathered}
T_{e}^{*}=\frac{3}{2} p\left|\phi_{s}^{*}\right|\left(\frac{\phi_{f}}{L_{d}} \sin \delta^{*}+\frac{\left|\phi_{s}^{*}\right|}{2}\left(\frac{1}{L_{q}}-\frac{1}{L_{d}}\right) \sin 2 \delta^{*}\right) \\
\tan \left(\phi_{i \phi}^{*}\right)=\frac{T_{e}^{*}}{\frac{3 p\left|\phi_{s}^{*}\right|}{2}\left(\frac{\left|\phi_{s}^{*}\right|}{L_{d}} \cos ^{2} \delta^{*}+\frac{\left|\phi_{s}^{*}\right|}{L_{q}} \sin ^{2} \delta^{*}-\frac{\phi_{f}}{L_{d}} \cos \delta^{*}\right)} \\
\left|i_{s}^{*}\right|=\frac{T_{e}^{*}}{\frac{3}{2} p\left|\phi_{s}^{*}\right| \sin \left(\phi_{i \phi}^{*}\right)}
\end{gathered}
$$

根据上述分析, 由式 (19) 求出 $\delta^{*}$, 代入式 (20) 求出 $\phi_{i \phi}^{*}$, 再代入式 $(21)$ 求出最优电流矢量参考值 $\left|i_{s}^{*}\right|$ 。 根据式（6），(7)，(10）得:

$$
\left|i_{s}\right|=\frac{T_{e}^{*}}{\frac{3}{2} p\left|\phi_{s}^{*}\right| \sin \left(\phi_{i \phi c}\right)}
$$

$$
\left|\phi_{s}\right|=\sqrt{\left|\phi_{s}^{*}\right|^{2}+\left(\frac{\Delta R_{s}\left|i_{s}\right|}{\omega}\right)^{2}-\frac{4 T_{e}^{*}}{3 p \omega} \Delta R_{s}}
$$

根据式（6），（7），(16）得:

$$
\phi_{i \phi c}=\sin ^{-1}\left(\frac{T_{e c}}{\frac{3}{2} p\left|\phi_{s c}\right| i_{s c} \mid}\right)=\sin ^{-1}\left(\frac{T_{e}^{*}}{\frac{3}{2} p\left|\phi_{s}^{*}\right|\left|i_{s}\right|}\right)
$$

综上所述, 可以得知, 定子电阻变化会引起负载角变 化, 进而引起定子磁链和电流矢量之间夹角变化, 从而导 致电流矢量发生变化, 电流矢量的变化引起定子磁链估计 值与真实值之间的差异, 这种偏差引起定子磁链发生改变, 导致DTC系统性能变差。电机在低速运行时由于输入电压 小, 定子电阻压降明显影响系统控制性能, 从而导致电机 的不稳定运行。

本文基于PI控制原理, 根据定子电阻变化会引起定子 电流发生对应变化原则, 对定子电阻进行在线估计, 减小 因定子电阻的变化对磁链观测和电流带来不良影响, 提高 控制系统鲁棒性。定子电阻估计器的原理方框图如图3所 示。

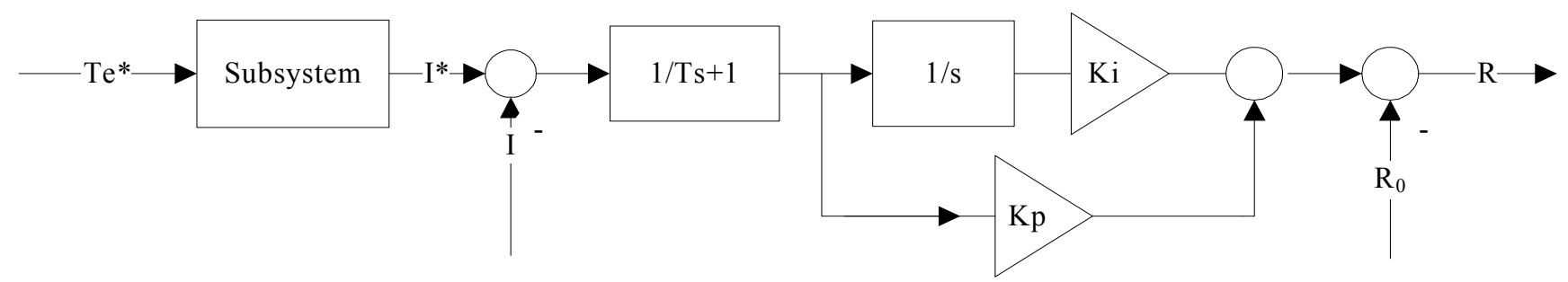

图3 PI定子电阻估计器的原理方框图。

PI电阻估计器公式为:

$$
\Delta R_{s}=k_{p} \Delta I_{s}+k_{i} \Delta I_{s} / s
$$

其中 $k_{p}$ 为 $\mathrm{PI}$ 估计器的比例; $k_{i}$ 为积分增益。

\section{4. 仿真实验}

为验证上述定子电阻估计器的有效可行性, 利用 Matlab/Simulink仿真工具进行系统仿真研究。电机参数 如表1所示。根据图3, 定子电阻仿真模块如图4所示。
表1 电机特征参数。

\begin{tabular}{llll}
\hline 参数 & 参数表示 & 数值 & 单位 \\
\hline 定子电阻 & $R s$ & 1.2 & $\Omega$ \\
电感 & $L$ & 8.5 & $\mathrm{mH}$ \\
转子磁链 & $\psi_{f}$ & 0.175 & $\mathrm{~Wb}$ \\
极对数 & $P$ & 4 & \\
额定电压 & $U$ & 300 & $\mathrm{~V}$ \\
转动惯量 & $J$ & 0.0008 & $\mathrm{~kg} . \mathrm{m}^{2}$ \\
\hline
\end{tabular}




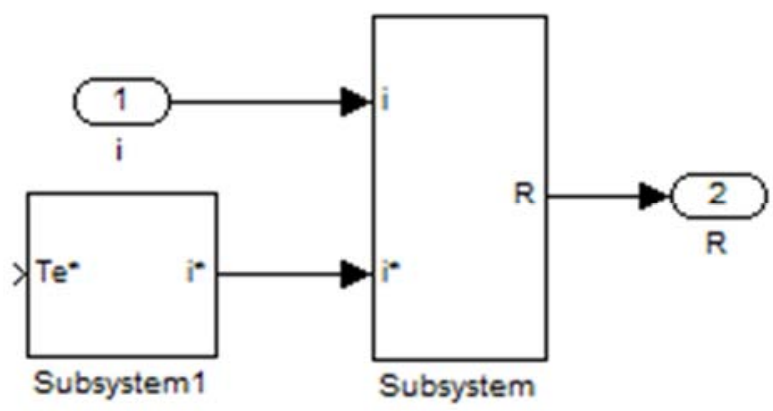

图4 定子电阻估计器模块。

设定电机运行时速度为 $600 \mathrm{r} / \mathrm{min}$, 传统DTC与改进电阻后的控制系统的定子磁链图、转矩图、三相电流图见图5 所示。

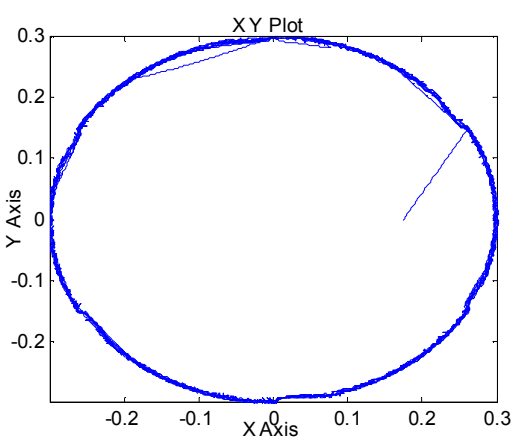

a）传统DTC定子磁链图

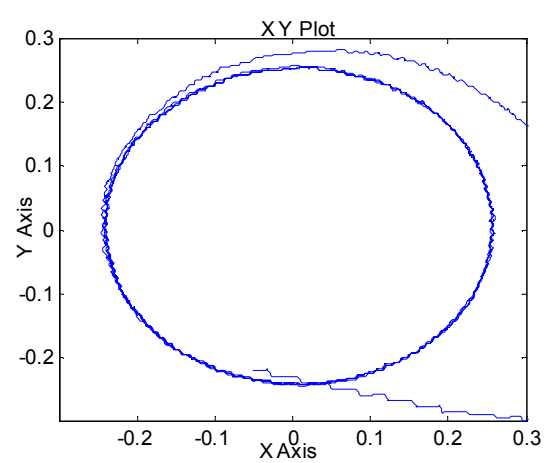

b) 改进定子电阻定子磁链图

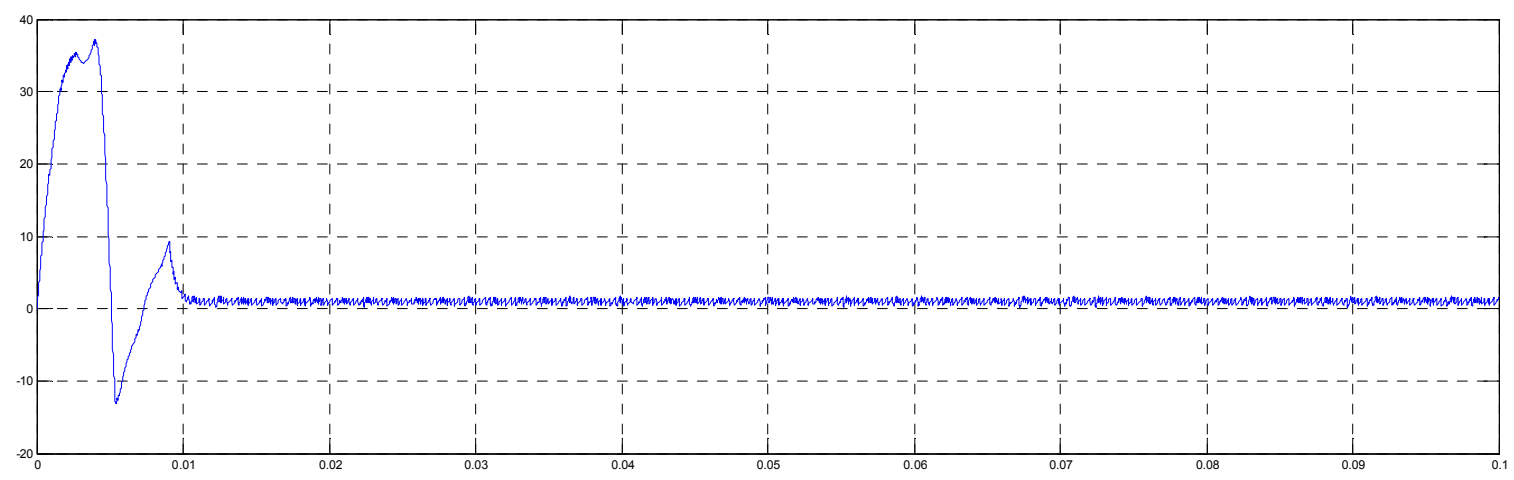

c) 传统DTC转矩波形图 


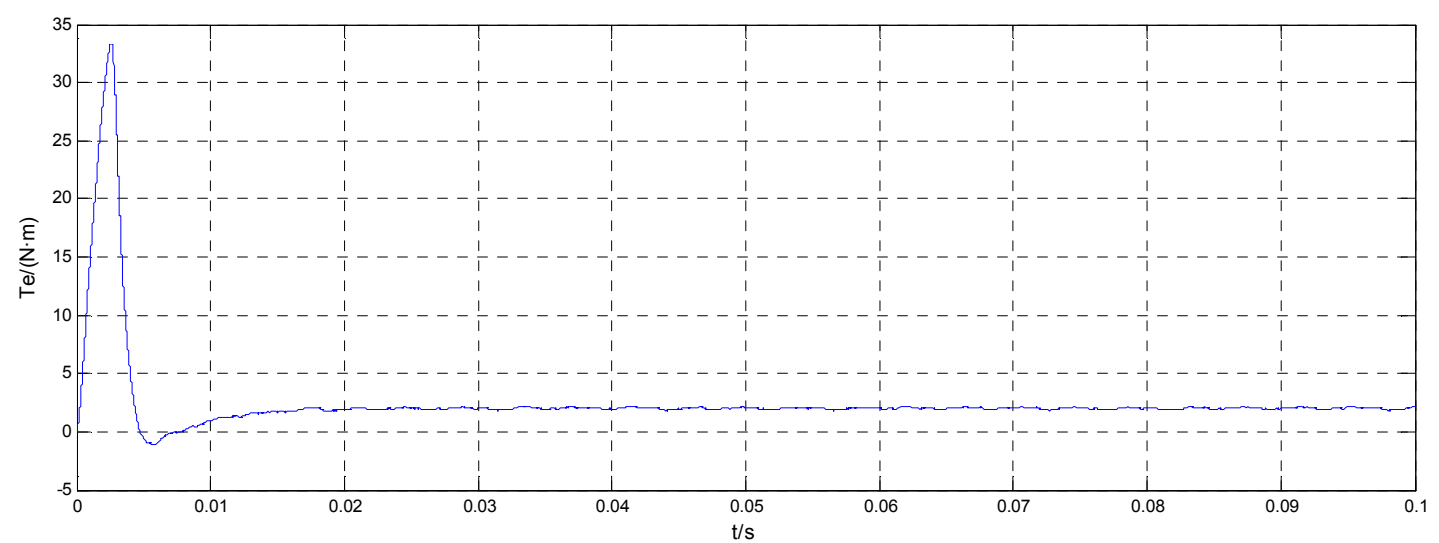

d）改进定子电阻波形图

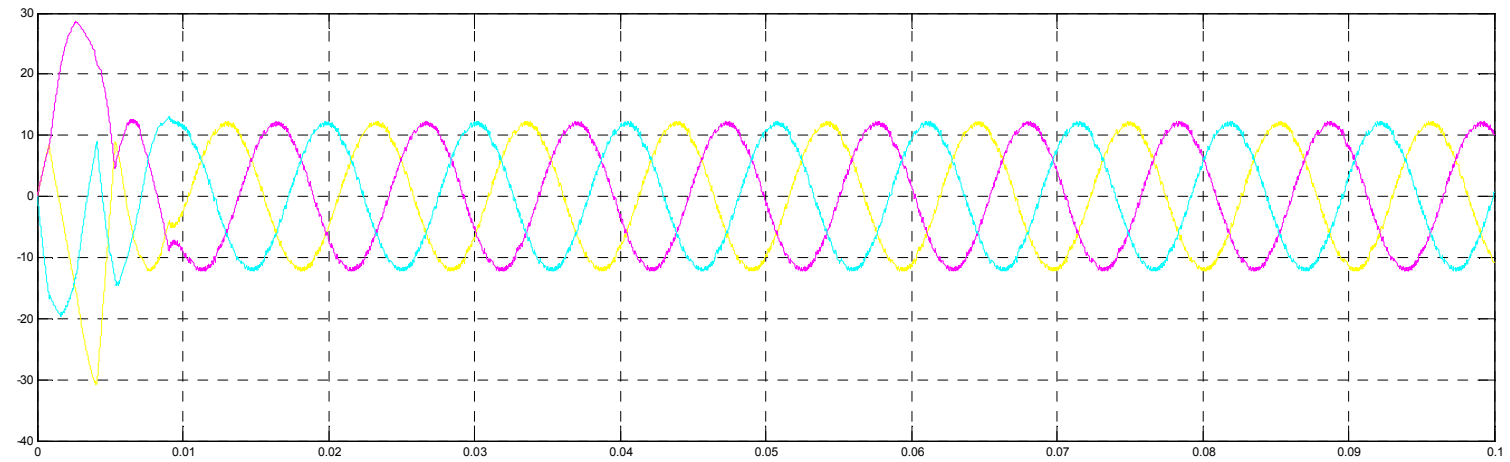

e) 传统DTC三相电流波形图

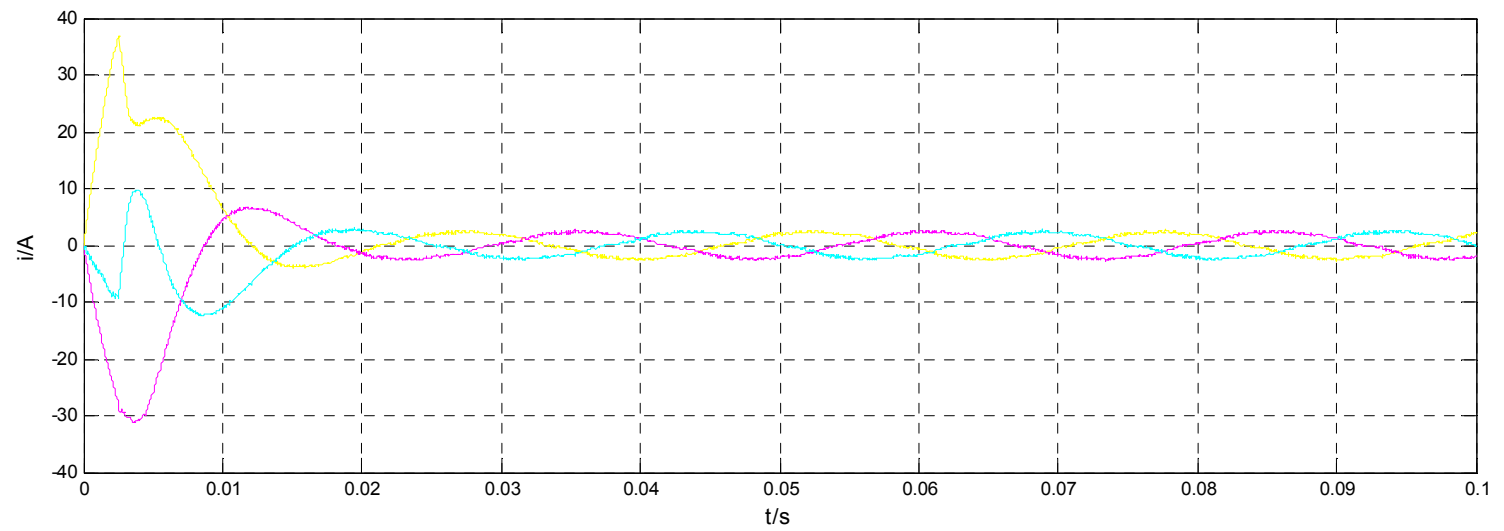

f）改进后三相电流波形图

图5 传统DTC与改进后系统对比图。

仿真选取控制系统运行时间为 $0.1 \mathrm{~s}$, 对比磁链波形图, 可以看到定子磁链对定子电阻变化敏感性降低, 系统鲁棒 性得到提升。对比转矩图, 传统DTC在 $0-0.01 \mathrm{~s}$ 转矩脉动大, 基于定子电阻估计器在 $0-0.01 \mathrm{~s}$ 脉动减小, 明显提高了系 统鲁棒性。对比三相电流, 系统运行初期脉动减小, 系统 响应时间减小。

经过对比传统DTC与改进后的仿真图相比较, 定子电 阻的影响导致定子磁链在电机运行初期发生的明显偏差
已经得到较好的修正, 磁链脉动减小, 稳态性明显提升, 电磁转矩脉动较传统DTC系统减小。

\section{5. 结论}

系统在运行过程中电机温度会发生变化进而引起定 子电阻的变化, 定子电阻的改变影响定子磁链观测器的准 确性, 使得系统控制性能变差。本文从一种新的角度上分 析研究定子电阻变化对定子磁链带来的影响, 用电流的变 
化来观测定子电阻变化, 消除电机在运行时由于定子电阻 变化引起的转矩和磁链幅值误差, 改善系统控制性能, 提 高系统鲁棒性。此外, 利用Matlab/Simulink搭建系统数 学模型进行仿真实验, 仿真结果表明该方法的有效性。

\section{致谢}

本文为国家自然科学基金（5150748）的阶段性成果 之一。

\section{参考文献}

[1] 赵利. 同步电动机励磁电流计算与用电系统功率因数提高 [J]. 河北工业科技, 2011, 01:26-29.

[2] 唐丽婵, 齐亮. 永磁同步电机的应用现状与发展趋势 $[\mathrm{J}]$. 装 备机械, 2011(1) :7-12.

[3] 张苏英, 李林静, 刘慧贤等. 基于滑模自适应的永磁同步电 动机无传感器控制 [J]. 河北科技大学学 报, 2016, 37 (4) : 1-2.

[4] 万中奇. 永磁同步发电机预测直接转矩控制策略研究 [C]. 浙江大学. 2013. 3:6-7.

[5] 李夙. 异步电动机直接转矩控制 [M]. 北京: 机械工业出版 社, 1993.

[6] 阮毅, 张晓华等. 感应电动机按定子磁场定向控制 $[\mathrm{J}]$. 电工 技术学报, 2003, 18(2): 1-4.

７］张春朋, 林飞, 宋文超. 基于定子电流矢量定向的异步电机 转子磁链估计器及其应用研究 $[J]$. 中国电机工程学报, 2003, 23 (8) : 155-158

[8] Abbou A, Mahmoudi H, and Elbacha A, "The effect of stator resistance variation on DTFC of induction motor and its compensation, " in Proc. 14th IEEE Int. Conf. Electron. Circuits Syst., Dec. 2007: 894-898.

[9] Rahman M, Zhong L. Prpblems of Stator Flux Oriented Torque Controllers for the IPM. Proceedings of the Thirs International PEMC Conference, 2000, 15 (18): 342-345.
[10] Vasic V.A stator resistance estimation scheme for speed sensorless rptpr flux oriented induction motor drives [J]. IEEE Trans. on Energy Conversion, 2003, 18(4) : 476-483.

[11] 陈硕, 山田英二, 感应电机无速度传感器矢量控制系统的定 子电阻在线辨识 $[J]$. 中国电机工程学报 2003, 23 (2) : 88-92.

[12] 王涣刚, 徐文立, 杨耕. 感应电动机定子磁链与转矩解耦自 适应控制 [J]. 中国电机工程学报, 2004, 24 (12)：171-175.

[13] 吴玉香, 胡跃明. 二阶动态滑模控制在移动机械臂输出跟踪 中的应用 [J]. 控制理论与应用, 2006, 23 (3) :411420.

[14] Man L. Fast terminal sliding mode control design for nonlinear dynamical systems [J]. IEEE Trans. Circ. Sys., 2002, 49(2) : 261-264.

[15] Karanayil B, Rahman M F, Grantham C. Stator and rotor resistance observers for induction motor drive using fuzzy logic and artifical neural networks [J]. IEEE Trans. on Energy Conversion, 2005, 20(4) : 771-780.

[16] Karanayil B, Rahman M F, Grantham C. On-line stator and rotor resistance estimation scheme for vector con-trolled induction motor drive using artificial neural networks [C]. Proceedings of IAS, Texas, USA, 2003, 1: 132-139.

[17] Elbuluk M E, Liu Tong, Husain I. Neural-network-based model reference adaptive systems for high-performance motor drives and motion controls [J]. IEEE Trans. on Industry Applications, 2002, 38(3) : 879-886.

[18] 吕伟杰, 刘鲁源. 小波网络在直接转矩控制定子电阻辨识中 的应用 [J] 中国电机工程学报, 2004, 24 (4) : 116-119.

[19] 牛慧文. 永磁同步电机直接转矩控制系统的仿真研究 [C]. 太原理工大学, 2012. 5: 9-10.

[20] 袁雷, 胡冰新, 魏克音等. 现代永磁同步电机控制原理及 MATLAB仿真 [M]. 北京, 北京航空航天大学出版社, 2016: 95-97.

[21] 王䒯. 永磁同步电机直接转矩控制系统的研究 [C]. 南京航 空航天大学, 2012. 1: 4-9. 